\title{
Risk factors for locoregional recurrence in patients with pathologic T3N0 rectal cancer with negative resection margin treated by surgery alone
}

\author{
Jong Yun Baek ${ }^{1}$, Jeong II Yu' ${ }^{1}$, Hee Chul Park', Doo Ho Choi ${ }^{1}$, Gyu Sang Yoo', \\ Won Kyung Cho', Woo-Yong Lee², Seong Hyeon Yun², Yong Beom Cho², Yoon Ah Park², Hee Cheol Kim² \\ 'Department of Radiation Oncology, Samsung Medical Center, Sungkyunkwan University School of Medicine, Seoul, Korea \\ ${ }^{2}$ Department of General Surgery, Samsung Medical Center, Sungkyunkwan University School of Medicine, Seoul, Korea
}

Purpose: This study aimed to identify prognostic factors for locoregional recurrence (LRR) in pT3NO rectal cancer patients who were treated with surgery alone and had negative resection margin including circumferential resection margin (CRM) for optimal indication of adjuvant radiotherapy.

Materials and Methods: We reviewed patients with pT3NO rectal cancer who were treated via upfront surgery and had no other adjuvant treatment from January 2003 to December 2012. In total, 122 patients who had negative resection margin including negative CRM were included in the analysis.

Results: The median follow-up period after surgery was 60 months (range, 3 to 161 months). During this time, 6 patients (4.9\%) experienced LRR at the anastomotic site (4 patients), and regional lymphatic area (2 patients). The estimated 5 -year rates of overall survival, recurrence-free survival, and LRR-free survival were $96.7 \%, 84.6 \%$, and $94.0 \%$, respectively. Multivariate analysis showed that level of tumor $\leq 5 \mathrm{~cm}$ was a significant prognostic factor for LRR-free survival (LRRFS) ( $p=0.04$; hazard ratio $=7.08$; $95 \%$ confidence interval, 1.06-47.30). Patients with level of tumor $\leq 5 \mathrm{~cm}$ had an estimated 5-year LRRFS of $66.8 \%$, which was much higher than $2.3 \%$ in patients with level of tumor $>5 \mathrm{~cm}$. There was no significant factor for recurrence-free survival or overall survival.

Conclusion: In T3NO rectal cancer, adjuvant chemoradiotherapy should be recommended in patients with level of tumor $\leq 5 \mathrm{~cm}$ for better local control. However, in patients with pT3NO disease, negative resection margin, and level of tumor $>5 \mathrm{~cm}$, adjuvant chemoradiotherapy should be carefully suggested.

Keywords: Rectal neoplasms, T3NO, Surgery alone

\section{Introduction}

Colorectal cancer is the third most commonly diagnosed malignancy and the fourth leading cause of cancer deaths

Received 02 April 2019, Revised 15 April 2019, Accepted 26 April 2019.

Correspondence: Jeong II Yu, Department of Radiation Oncology, Samsung Medical Center, Sungkyunkwan University School of Medicine, 81 Irwon-ro, Gangnam-gu, Seoul 06351, Korea. Tel: +82-2-3410-9598, Fax: +82-2-3410-2619, E-mail: jeongil.yu@samsung.com (https://orcid.org/0000-0002-2009-7263)

Correspondence: Hee Cheol Kim, Department of General Surgery, Samsung Medical Center, Sungkyunkwan University School of Medicine, 81 Irwon-ro, Gangnam-gu, Seoul 06351, Korea. Tel: +82-2-3410-1655, Fax: +82-2-3410-6980, E-mail: hc111.kim@samsung.com (https://orcid.org/0000-0003-0385-9681)

(c) This is an Open Access article distributed under the terms of the Creative Commons Attribution Non-Commercial License (http://creativecommons.org/ licenses/by-nc/4.0/) which permits unrestricted non-commercial use, distribution, and reproduction in any medium, provided the original work is properly cited.

www.e-roj.org 
worldwide, accounting for about 1.4 million new cases per year. Surgical resection is a standard treatment modality for non-metastatic rectal cancer. However, in locally advanced rectal cancer, curative surgery alone resulted in high rates of locoregional recurrence $(L R R)[1,2]$. To reduce $L R R$, neoadjuvant or adjuvant radiotherapy (RT) was recommended in locally advanced rectal cancers (T3/T4 or $\mathrm{N}+$ tumors) $[3,4]$. Although RT decreases LRR rates, it is also related with increased treatment-associated adverse events including fecal incontinence and sexual dysfunction [5].

After the introduction of total mesorectal excision (TME) which reduces LRR greatly compared to conventional surgery, there is controversy about the necessity of RT in patients with pathologic T3N0 (pT3No) disease which has a relatively low LRR rate and good prognosis [6]. Several retrospective studies reported an $L R R$ of $<10 \%$ in patients with minimal pT3NO rectal cancer without adjuvant treatment after TME $[7,8]$.

Currently, neoadjuvant chemoradiotherapy (CCRT) followed by TME in locally advanced rectal cancer is considered as standard management. In this era, it is much controversial that adjuvant RT is necessary, when clinically T2 or early T3NO rectal cancer is treated via upfront surgery with TME and the pathologic stage is T3NOMO with sufficient circumferential resection margin (CRM) [7,9-12]. In those cases, adjuvant RT only for patients with high-risk features should be considered to reduce unnecessary exposure to $\mathrm{RT}$ and/or RT-related toxicities. Risk adaptive customized treatment decision according to the prognostic factors of LRR is essential in these patients.

Against this background, we conducted the present study to identify optimal indications of adjuvant RT evaluating prognostic factors on LRR in PT3NO rectal cancer patients who were treated with standardized surgery-low anterior resection (LAR) or abdominoperineal resection (APR)-with TME and had negative resection margin and negative $C R M$, defined as more than $1 \mathrm{~mm}$ distance between the tumor and CRM.

\section{Materials and Methods}

Patients who underwent surgery for rectal cancer at the Samsung Medical Center in Korea from January 2003 to December 2012 were retrospectively reviewed. The inclusion criteria of the present study were as follows: (1) no preoperative therapy, (2) underwent LAR or APR with TME, (3) pathological T3NO rectal cancer without distant metastasis (DM), (4) negative resection margin and negative CRM (>1 $\mathrm{mm}$ ), (5) no adjuvant chemotherapy or RT, and (6) no other malignancy. All data were based on electronic medical records.
Of 416 patients with pT3N0 rectal cancer, 269 received adjuvant treatment such as chemotherapy alone or CCRT.

Level of tumor was given as the distance from the anal verge, measured on digital rectal examination by the surgeon, colonoscopy, or computed tomography/magnetic resonance imaging (MRI) scan. Length of tumor was given as the maximum tumor diameter. CRM $>1 \mathrm{~mm}$ was considered uninvolved.

During the follow-up period, all patients underwent regular follow-up including physical examination, tumor marker assessment, colonoscopy, and radiologic examination at intervals of 3 to 12 months. Lesions suspicious of recurrence were confirmed pathologically or by consecutive radiologic follow-up. Recurrence at the anastomotic site and tumor bed was defined as local recurrence, and recurrence at the regional lymphatic area as regional recurrence. LRR was defined as local recurrence or regional recurrence. Systemic disease spread outside the primary tumor basin was defined as DM.

Clinical outcomes analyzed were locoregional recurrencefree survival (LRRFS), recurrence-free survival (RFS), and overall survival (OS). LRRFS, RFS, and OS were calculated from the date of the primary surgery to the date of event detection or last follow-up. LRRFS was defined as the time to LRR at the first recurrence site with or without simultaneous DM. RFS and OS were defined as the time to first recurrence and death from any causes, respectively.

Data regarding patients' age, sex, operation, level of tumor, histology, tumor diameter, number of harvested lymph nodes, DRM, Iymphovascular invasion (LVI), perineural invasion, preoperative carcinoembryonic antigen (CEA), and preoperative carbohydrate antigen 19-9 (CA19-9) were collected for analysis.

Patients' characteristics were described using frequency tables. The association between categorical factors was analyzed using Fisher exact test or Pearson $\chi^{2}$ test. Univariate survival trends were compared using Kaplan-Meier curves and significant differences determined via the log-rank test. For multivariate modeling and examining the prognostic significance of the variables identified in the models, Cox proportional hazards regression was used. Variables which had $p<0.10$ on univariate analysis were forwardly selected for multivariate analyses. SPSS version 25 (IBM SPSS, Armonk, NY, USA) was used for all statistical analyses.

\section{Results}

\section{Patients}

Of 147 patients, there was no record about CRM in 11 patients, 
Table 1. Baseline characteristics of study patients $(n=122)$

\begin{tabular}{|c|c|}
\hline Variable & No. of patients (\%) \\
\hline \multicolumn{2}{|l|}{ Age (yr) } \\
\hline$<75$ & $90(73.8)$ \\
\hline$\geq 75$ & $32(26.2)$ \\
\hline \multicolumn{2}{|l|}{ Sex } \\
\hline Male & $67(54.9)$ \\
\hline Female & $55(45.1)$ \\
\hline \multicolumn{2}{|l|}{ Operation } \\
\hline LAR & $120(98.4)$ \\
\hline APR & $2(1.6)$ \\
\hline \multicolumn{2}{|l|}{ Level of tumor (cm) } \\
\hline$\leq 5$ & $17(13.9)$ \\
\hline$>5$ and $\leq 10$ & $67(54.9)$ \\
\hline$>10$ and $\leq 15$ & $38(31.1)$ \\
\hline \multicolumn{2}{|l|}{ Histology } \\
\hline ADC, well differentiated & $20(16.4)$ \\
\hline $\begin{array}{l}\text { ADC, moderately } \\
\text { differentiated }\end{array}$ & $99(81.1)$ \\
\hline Mucinous carcinoma & $3(2.5)$ \\
\hline \multicolumn{2}{|l|}{ Tumor diameter $(\mathrm{cm})$} \\
\hline$>4$ & $43(35.2)$ \\
\hline$\leq 4$ & $79(64.8)$ \\
\hline \multicolumn{2}{|l|}{ No. of harvested LN } \\
\hline$\leq 12$ & $25(20.5)$ \\
\hline$>12$ & $97(79.5)$ \\
\hline \multicolumn{2}{|l|}{ DRM (cm) } \\
\hline$\leq 2$ & $57(46.7)$ \\
\hline$>2$ & $65(53.3)$ \\
\hline \multicolumn{2}{|l|}{ LVI } \\
\hline Yes & $6(4.9)$ \\
\hline No & $115(94.3)$ \\
\hline Unknown & $1(0.8)$ \\
\hline \multicolumn{2}{|l|}{ PNI } \\
\hline Yes & $8(6.6)$ \\
\hline No & $103(84.4)$ \\
\hline Unknown & $11(9.0)$ \\
\hline \multicolumn{2}{|l|}{ CEA (ng/mL) } \\
\hline$\leq 5$ & $93(76.2)$ \\
\hline$>5$ & $26(21.3)$ \\
\hline Unknown & $3(2.5)$ \\
\hline
\end{tabular}

LAR, low anterior resection or Hartmann's operation; APR, abdominoperineal resection; $A D C$, adenocarcinoma; DRM, distal resection margin; $L N$, lymph node; $L V I$, lymphovascular invasion; $\mathrm{PNI}$, perineural invasion; CEA, carcinoembryonic antigen.

and 8 patients had positive CRM. Six were lost to follow-up immediately after surgery. Finally, the records of 122 patients were carefully reviewed and analyzed in this study.

The median age was 67 years (range, 38 to 89 years). The male to female ratio was 1.22:1. Preoperative serum CEA levels were recorded in 119 patients (97.5\%) with elevated CEA (>5 $\mathrm{ng} / \mathrm{mL}$ ) in 26 patients (21.3\%). For the analysis, level of tumor

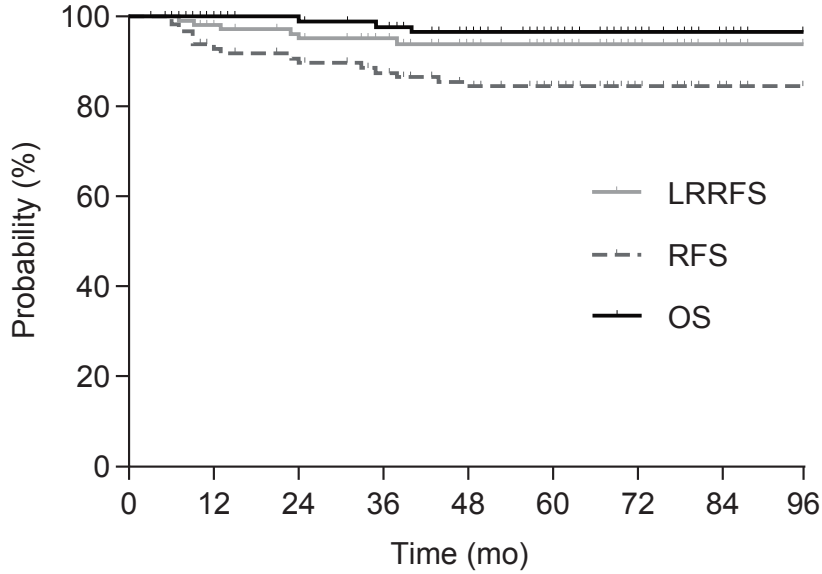

Fig. 1. Kaplan-Meier curves for locoregional recurrence-free survival (LRRFS), recurrence-free survival (RFS), overall survival (OS) for all included patients. The 5-year LRRFS, RFS, and OS were $94.0 \%, 84.6 \%$, and $96.7 \%$, respectively.

was divided by $5 \mathrm{~cm}$, which meant lower rectal cancer, and in 17 patients (13.9\%), the level of tumor was $5 \mathrm{~cm}$ or lower. All patients had negative resection margins, but 57 patients (46.7\%) had insufficient DRM $(\leq 2 \mathrm{~cm})$ and 15 patients $(12.3 \%)$ had an insufficient number of harvested lymph nodes $(<12)$ (Table 1).

\section{Clinical outcomes}

The median follow-up period was 60 months (range, 3 to 161 months). During the follow-up period, 6 patients (4.9\%) developed LRR, 10 patients (9.1\%) developed DM, and 3 patients (2.5\%) died.

Among the 6 patients who showed LRR, 5 (83.3\%) experienced LRR within 2 years after surgical resection. The location of the primary tumors in those patients were $1 \mathrm{~cm}$ in 1 patient, $4 \mathrm{~cm}$ in 3 patients, $6 \mathrm{~cm}$ in 1 patient, and $9 \mathrm{~cm}$ in 1 patient from the anal verge. The anastomotic site was the most common site of LRR ( 4 of 6 patients). Otherwise, in one patient, recurrence developed on the presacral area at the S2 level, and in the other, on the presacral area of the coccyx and internal iliac lymph nodes at the S3 level.

Among the ten patients who showed DM, 6 patients (60\%) experienced DM within 2 years after surgical resection. The lung was the most common area of DM (4 patients), followed by the common iliac LNs (2 patients), liver (2 patients), mediastinal LN (1 patient), and ovary (1 patient).

The estimated 5-year LRRFS, RFS, and OS were 94.0\%, $84.6 \%$, and $96.7 \%$, respectively (Fig. 1). 
Table 2. Prognostic factors of LRRFS, RFS, and OS in univariate analysis

\begin{tabular}{|c|c|c|c|c|c|c|c|}
\hline \multirow{2}{*}{ Variable } & \multirow{2}{*}{$\begin{array}{c}\text { No. of } \\
\text { patients }\end{array}$} & \multicolumn{2}{|c|}{ 5-yr LRRFS } & \multicolumn{2}{|c|}{ 5-yr RFS } & \multicolumn{2}{|c|}{$5-y r O S$} \\
\hline & & $\%$ & $p$-value & $\%$ & p-value & $\%$ & $p$-value \\
\hline \multicolumn{8}{|l|}{ Age (yr) } \\
\hline$<75$ & 90 & 97.3 & $0.01^{*}$ & 87.6 & 0.096 & 98.7 & $0.04^{*}$ \\
\hline$\geq 75$ & 32 & 84.1 & & 75.5 & & 88.1 & \\
\hline \multicolumn{8}{|l|}{ Level of tumor $(\mathrm{cm})$} \\
\hline$\leq 5$ & 17 & 66.8 & $<0.001^{*}$ & 55.7 & $0.01^{*}$ & 90.9 & 0.18 \\
\hline$>5$ & 105 & 97.7 & & 88.1 & & 97.5 & \\
\hline \multicolumn{8}{|l|}{ No. of harvested LN } \\
\hline$\leq 12$ & 25 & 81.3 & $0.01^{*}$ & 71.8 & 0.097 & 100 & 0.39 \\
\hline$>12$ & 97 & 97.5 & & 88.1 & & 96.0 & \\
\hline \multicolumn{8}{|l|}{ DRM (cm) } \\
\hline$\leq 2$ & 57 & 88.2 & $0.04^{*}$ & 87.6 & 0.25 & 94.3 & 0.32 \\
\hline$>2$ & 65 & 98.2 & & 81.1 & & 98.2 & \\
\hline \multicolumn{8}{|l|}{ LVI } \\
\hline Yes & 6 & 100 & 0.60 & 83.3 & 0.84 & 100 & 0.69 \\
\hline No & 115 & 94.7 & & 85.6 & & 96.6 & \\
\hline \multicolumn{8}{|l|}{ PNI } \\
\hline Yes & 8 & 100 & 0.55 & 72.9 & 0.33 & 100 & 0.67 \\
\hline No & 103 & 95.4 & & 87.7 & & 97.4 & \\
\hline \multicolumn{8}{|l|}{ CEA (ng/mL) } \\
\hline$\leq 5$ & 93 & 96.0 & 0.05 & 87.4 & 0.16 & 98.5 & 0.24 \\
\hline$>5$ & 26 & 85.0 & & 77.5 & & 94.4 & \\
\hline
\end{tabular}

LRRFS, locoregional recurrence-free survival; RFS, recurrence-free survival; OS, overall survival; DRM, distal resection margin; LN, lymph node; LVI, Iymphovascular invasion; PNI, perineural invasion; CEA, carcinoembryonic antigen; CA 19-9, carbohydrate antigen 19-9; NLR, neutrophil-to-lymphocyte ratio; LMR, lymphocyte-to-monocyte ratio. ${ }^{*} p<0.05$.

\section{Prognostic factors}

All possible prognostic factors were evaluated by univariate analysis. Age $\geq 75$ years, level of tumor $\leq 5 \mathrm{~cm}, \mathrm{DRM} \leq 2 \mathrm{~cm}$ were significant poor prognostic factors for LRRFS. Both CEA $>5 \mathrm{ng} / \mathrm{mL}$ and CA19-9 >30 ng/mL had borderline significance

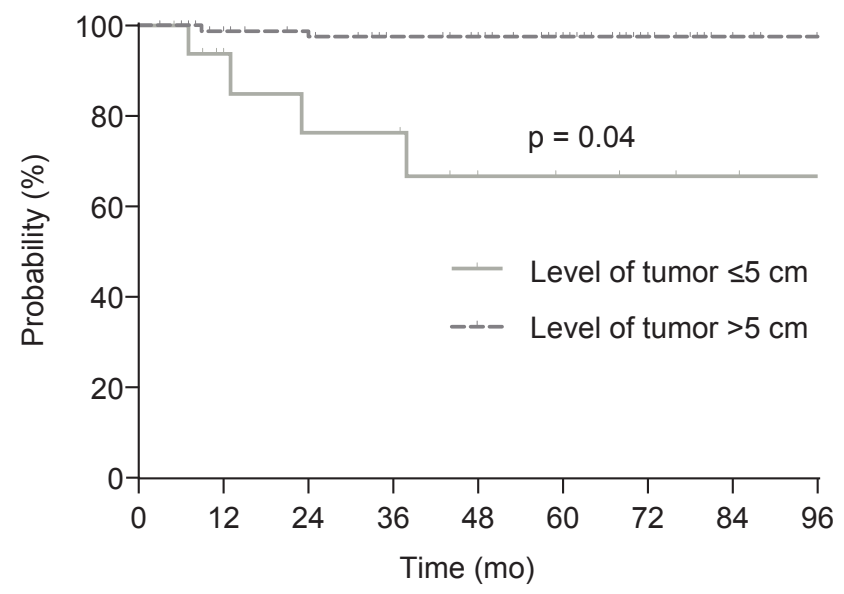

Fig. 2. Locoregional recurrence-free survival (LRRFS) according to level of tumor. The 5 -year LRRFS was $66.8 \%$ in patients with level of tumor $\leq 5 \mathrm{~cm}$ and $97.7 \%$ in patients with level of tumor $>5 \mathrm{~cm}$ (p $\leq 0.001)$.
( $p=0.05)$, and the number of harvested lymph nodes $<12$ was not a significant factor, but had a tendency of higher LRR ( $p=$ 0.098). Level of tumor $\leq 5 \mathrm{~cm}$ also had a significant influence on RFS. For OS, age $\geq 75$ years was the only significant prognostic factor (Table 2).

In the multivariate analysis, CA19-9 was not checked in $>20 \%$ of patients, and so was not included. For LRRFS, level of tumor $\leq 5 \mathrm{~cm}$ was the only significant factor $(p=0.04$; hazard ratio $=7.08 ; 95 \%$ confidence interval, 1.06-47.30). Seventeen patients (13.9\%) with level of tumor $\leq 5 \mathrm{~cm}$ had an estimated 5 -year LRRFS of $66.8 \%$. By contrast, the estimated 5 -year LRRFS was only $2.3 \%$ in 105 patients (86.0\%) who had level of tumor $>5 \mathrm{~cm}$ (Fig. 2).

For RFS and OS, there was no significant factor (Table 3).

\section{Discussion and Conclusion}

In this retrospective study evaluating 122 patients with PT3NO rectal cancer who had negative CRM and no adjuvant treatment, 5-year LRRFS, 5-year RFS, and 5-year OS were 94.0\%, 84.6\%, and 96.7\%, respectively. For LRRFS, level of tumor $\leq 5 \mathrm{~cm}$ was found to be the only significant risk factor 
Table 3. Prognostic factors of LRRFS and RFS in multivariate analysis

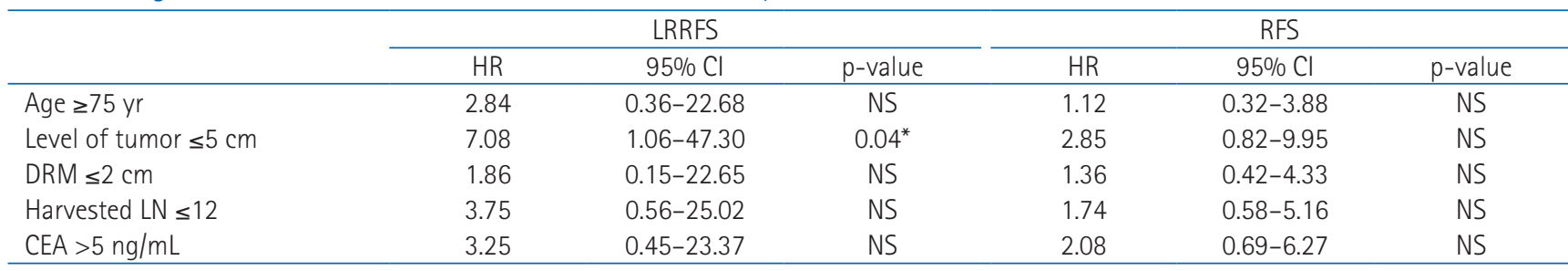

LRRFS, locoregional recurrence-free survival; RFS, recurrence-free survival; $\mathrm{HR}$, hazard ratio; $\mathrm{Cl}$, confidence interval; DRM, distal resection margin; LN, lymph node; CEA, carcinoembryonic antigen; NS, not significant.

${ }^{*} p<0.05$.

on multivariate analysis and patients with level of tumor $\leq 5$ $\mathrm{cm}$ had an 66.8\% 5-year LRRFS. In contrast, the 5-year LRRFS of patients who had level of tumor $>5 \mathrm{~cm}$ was only $97.7 \%$. Most LRRs (5 of 6 patients) occurred within 2 years after surgical resection.

In locally advanced rectal cancer, the LRR rate was very high after surgical resection alone [1,2]. To improve local control, neoadjuvant or postoperative CCRT became the standard management in locally advanced rectal cancer [3,4]. However, with the introduction of TME, LRRs after surgery alone much decreased in locally advanced rectal cancer [6]. In the era of TME, pT3NO rectal cancer which had the most favorable outcome within the group of locally advanced rectal cancer also showed much-improved outcomes. Several retrospective studies reported LRR rates of 4\%-19\% in patients with pT3NO disease treated with TME and not irradiated, which was quite lower than in the pre-TME era $[8,9,12-14]$. Although RT improves local control, RT-related toxicity, such as gastrointestinal toxicity, genitourinary toxicity, and sexual dysfunction, could occur [5]. Thus, it is controversial that postoperative CCRT is necessary for pT3NO rectal cancer with low risk features [11].

According to the National Comprehensive Cancer Network (NCCN) guidelines, neoadjuvant CCRT followed by TME is the standard management in locally advanced rectal cancer. However, in the European Society for Medical Oncology guidelines, surgery without neoadjuvant CCRT could be performed in cT2-early T3 NO rectal cancer due to low risk features of the disease or other reasons [17]. If stage is confirmed pathologically as T3NO after upfront surgery, whether adjuvant CCRT is added or not is always a problem. To solve this problem, a selective strategy of adjuvant treatment is required, and several retrospective studies tried to identify risk factors for $L R R$.

In the era of TME, due to routine use of neoadjuvant or adjuvant treatment, there was limited data about pT3NO disease with no adjuvant treatment, and the risk factors associated with LRR were inconsistently reported probably due to the small number of patients and/or differences in various adjuvant treatment strategies of each institution. Merchant et al. [9] evaluated 95 patients with pT3NO disease without adjuvant therapy. The clinical outcomes were $12 \%$ and $75 \%$ for 5-year LRR rate and 5-year OS, respectively. In that study, only LVI was a significant factor for LRR on univariate analysis. Nissan et al. [8] reviewed 94 patients with T2-3 No rectal cancer treated with surgery alone. The local recurrence rate was $4.1 \%$ for T3N0 disease. On univariate analysis, LVI, and preoperative CEA $>5 \mathrm{ng} / \mathrm{mL}$ were significantly associated with LRR. In a study conducted by Zhu et al. [14], on the evaluation of 122 patients with pT3NO disease treated with surgery alone, lower tumor location was identified as a risk factor for LRR again. In the Norwegian rectal cancer study which evaluated 1,676 patients with pT3 rectal cancer with TME only, 890 patients had pT3NO disease. Among these patients, the 5-year LRR rate was $19.4 \%$ with $C R M \leq 1 \mathrm{~mm}$ compared with $11.1 \%$ for patients with CRM $>3 \mathrm{~mm}$ [13]. More recently, Wu et al. [12] evaluated 141 patients with pT3N0 rectal cancer who received adjuvant chemotherapy or CCRT. In that study, there was no surgery alone arm, but in the chemotherapy-only group, the 5 -year LRR rate was $18.3 \%$ and the 5 -year OS was $83.3 \%$ Preoperative CEA level, number of harvested lymph nodes, and perirectal fat infiltration were associated with RFS on multivariate analysis.

In our study, all included patients did not receive any adjuvant RT or chemotherapy as primary treatment. At the Samsung Medical Center, adjuvant treatment was delivered in patients with PT3NO disease based on clinicians' decision and patients' opinion, considering recognized risk factors of recurrence, comorbidities, or old age. Thus, in this study, only a few patients had well-known risk factors like CEA $>5 \mathrm{ng} / \mathrm{mL}$ (26 patients, 21.3\%), number of harvested lymph nodes $<12$ (25 patients, 20.5\%), poorly differentiated carcinoma (0 patients, 
0\%), LVI (6 patients, 4.9\%), and perineural invasion (8 patients, $6.6 \%)$. Furthermore, patients with positive resection margin or $\mathrm{CRM} \leq 1 \mathrm{~mm}$, in which adjuvant CCRT is considered essential because obviously worse outcome is expected, were excluded $[15,16]$. As a result, in our study, the 5-year LRRFS and 5-year RFS were $94.0 \%$ and $84.6 \%$, respectively, which were much better than in other studies.

Considering such low incidence of LRR, the result that on multivariate analysis all other factors except for level of tumor $\leq 5 \mathrm{~cm}$ were not significant not anymore was not surprising. However, even with good local control, the estimated 5-year LRRFS in patients with level of tumor $\leq 5 \mathrm{~cm}$ was over $30 \%$ which was unacceptably high. In these patients, adjuvant CCRT should be strongly recommended. On the other hand, the estimated 5-year LRR rate was just 2.3\% in 105 patients (86.0\%) with level of tumor $>5 \mathrm{~cm}$. Therefore, in patients with CRM $>1 \mathrm{~mm}$, level of tumor $>5 \mathrm{~cm}$, adjuvant CCRT should be carefully considered.

Other factors like age $\geq 75$ years, DRM $\leq 2 \mathrm{~cm}$, number of harvested lymph nodes $\leq 12, \mathrm{CEA}>5 \mathrm{ng} / \mathrm{mL}$, and CA19-9 $>30$ $\mathrm{ng} / \mathrm{mL}$ also should be assessed again in a larger study.

The main limitation of this study is that all data were retrospectively collected at a single institution, which was a general tertiary hospital where highly experienced medical staff treated a large volume of patients. At our institution, MRI was routinely performed for diagnostic workup on advanced rectal cancer, so most patients who received upfront surgery without neoadjuvant treatment had $\mathrm{T} 2$ or $\mathrm{T} 3 \mathrm{a}-\mathrm{b}$ disease and were node negative. Furthermore, patients with pT3NO rectal cancer, especially with pathologically high-risk features, received adjuvant treatment. Thus, the number of patients with PT3NO rectal cancer treated with surgery alone was very limited, and these patients, as mentioned above, mostly had low risk features. There is the need to assess whether risk factors in this highly selected group of patients are equally useful in the general group in future studies.

Also, some of patients had restricted description about CRM like $>1 \mathrm{~mm}$ or $>10 \mathrm{~mm}$. Considering that CRM is a well-known significant prognostic factor, more detailed information on CRM is required in future studies.

Despite limitations, we found that level of tumor $\leq 5 \mathrm{~cm}$ was associated with higher LRR rate in patients with pT3NO rectal cancer treated via surgery alone and CRM(-). Considering that the 5-year LRR rate was over 30\% in patients with level of tumor $\leq 5 \mathrm{~cm}$, adjuvant CCRT should be strongly recommended in these patients. In contrast, the 5-year LRR rate was below 5\% in patients with $\mathrm{CRM}(-)$, level of tumor $>5 \mathrm{~cm}$; thus, adjuvant
CCRT should be carefully suggested at the clinician's discretion.

\section{Conflict of Interest}

No potential conflict of interest relevant to this article was reported.

\section{References}

1. McDermott FT, Hughes ES, Pihl E, Johnson WR, Price $A B$. Local recurrence after potentially curative resection for rectal cancer in a series of 1008 patients. Br J Surg 1985;72:34-7.

2. Minsky BD, Mies C, Recht A, Rich TA, Chaffey JT. Resectable adenocarcinoma of the rectosigmoid and rectum. I. Patterns of failure and survival. Cancer 1988;61:1408-16.

3. Krook JE, Moertel CG, Gunderson LL, et al. Effective surgical adjuvant therapy for high-risk rectal carcinoma. N Engl J Med 1991;324:709-15.

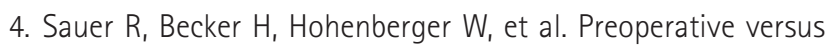
postoperative chemoradiotherapy for rectal cancer. N Engl J Med 2004;351:1731-40.

5. Lai LL, Fuller CD, Kachnic LA, Thomas CR Jr. Can pelvic radiotherapy be omitted in select patients with rectal cancer? Semin Oncol 2006;33(6 Suppl 11):S70-4.

6. Heald RJ, Ryall RD. Recurrence and survival after total mesorectal excision for rectal cancer. Lancet 1986;1:1479-82.

7. Enker WE, Thaler HT, Cranor ML, Polyak T. Total mesorectal excision in the operative treatment of carcinoma of the rectum. J Am Coll Surg 1995;181:335-46.

8. Nissan A, Stojadinovic A, Shia J, et al. Predictors of recurrence in patients with T2 and early T3, N0 adenocarcinoma of the rectum treated by surgery alone. J Clin Oncol 2006;24:407884.

9. Merchant NB, Guillem JG, Paty PB, et al. T3NO rectal cancer: results following sharp mesorectal excision and no adjuvant therapy. J Gastrointest Surg 1999;3:642-7.

10. Willett CG, Badizadegan K, Ancukiewicz M, Shellito PC. Prognostic factors in stage T3NO rectal cancer: do all patients require postoperative pelvic irradiation and chemotherapy? Dis Colon Rectum 1999;42:167-73.

11. Wo JY, Mamon HJ, Ryan DP, Hong TS. T3NO rectal cancer: radiation for all? Semin Radiat Oncol 2011;21:212-9.

12. Wu JX, Wang $Y$, Chen N, Chen LC, Bai PG, Pan JJ. In the era of total mesorectal excision: adjuvant radiotherapy may be unnecessary for pT3NO rectal cancer. Radiat Oncol 2014;9:159.

13. Eriksen MT, Wibe A, Haffner J, Wiig JN; Norwegian Rectal Cancer Group. Prognostic groups in 1,676 patients with T3 
rectal cancer treated without preoperative radiotherapy. Dis Colon Rectum 2007;50:156-67.

14. Zhu J, Xu Y, Gu W, et al. Adjuvant therapy for T3NO rectal cancer in the total mesorectal excision era-identification of the high risk patients. Radiat Oncol 2010;5:118.

15. Peeters KC, Marijnen CA, Nagtegaal ID, et al. The TME trial after a median follow-up of 6 years: increased local control but no survival benefit in irradiated patients with resectable rectal carcinoma. Ann Surg 2007;246:693-701.
16. Sebag-Montefiore D, Stephens RJ, Steele R, et al. Preoperative radiotherapy versus selective postoperative chemoradiotherapy in patients with rectal cancer (MRC CR07 and NCIC-CTG C016): a multicentre, randomised trial. Lancet 2009;373:811-20.

17. Glynne-Jones R, Wyrwicz L, Tiret E, et al. Rectal cancer: ESMO Clinical Practice Guidelines for diagnosis, treatment and follow-up. Ann Oncol 2017;28(suppl_4):iv22-iv40. 